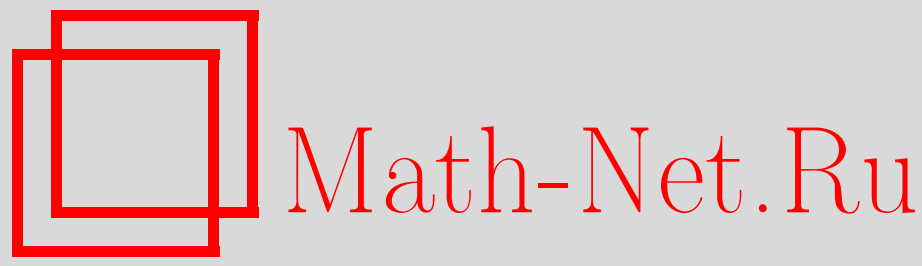

В. К. Ойконому, Преобразование обращения температуры в моделях калибровочного бозона Хиггса, ТMФ, 2009, том 159, номер 1, 109-130

DOI: https://doi.org/10.4213/tmf6336

Использование Общероссийского математического портала Math-Net.Ru подразумевает, что вы прочитали и согласны с пользовательским соглашением http://www.mathnet.ru/rus/agreement

Параметры загрузки:

IP: 54.166 .219 .16

26 апреля 2023 г., $17: 33: 10$

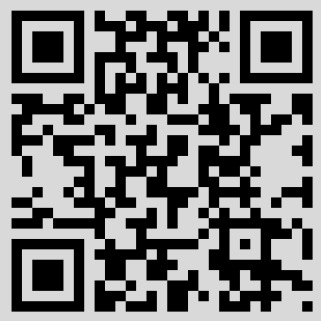




\title{
ПРЕОБРАЗОВАНИЕ ОБРАЩЕНИЯ ТЕМПЕРАТУРЫ В МОДЕЛЯХ КАЛИБРОВОЧНОГО БОЗОНА ХИГГСА
}

\begin{abstract}
Исследовано преобразование обращения температуры $R \rightarrow 1 / T$ для эффективного потенциала при конечной температуре в $N=1$ пятимерной суперсимметричной $S U(3)_{\mathrm{c}} \times S U(3)_{\mathrm{w}}$-модели на орбифолде $S^{1} / Z_{2}$. При значении параметра вильсоновской линии $\alpha=1\left(S U(2)_{\mathrm{L}}\right.$ при этом нарушена до $\left.U^{\prime}(1)\right)$ показано, что эффективный потенциал содержит симметричную и антисимметричную части относительно преобразования $\xi \rightarrow 1 / \xi, \xi=R T$. При $\alpha=0$ (в этом случае симметрия $S U(2)_{\mathrm{L}}$ сохраняется) найдено, что единственный вклад в эффективный потенциал, нарушающий симметрию обращения температуры, определяется фермионами в фундаментальном представлении калибровочной группы с $Z_{2}$ четностями $(+,+)$ или $(-,-)$. Этот результат представляет интерес, поскольку из него следует, что эффективный потенциал в объемлющем пространстве, отвечающий моделям с фундаментальными фермионами, локализованными в фиксированной орбифолдной точке (и моделям без фундаментальных фермионов в объемлющем пространстве), инвариантен относительно преобразования обращения температуры.
\end{abstract}

Ключевые слова: дополнительные размерности, симметрия обращения температуры, регуляризация с помощью дзета-функции, унификация, компактификация на орбифолды.

\section{1. ВВЕДЕНИЕ}

Дуальности имеют важное значение в квантовых теориях поля, особенно в эффективных теориях, поскольку выводы, следующие из дуальностей, выходят за рамки предсказаний, следующих из экспериментально доступной области или из теории возмущений. Симметрия обращения температуры, выражаемая преобразованием $R \rightarrow 1 / T$, связывает вакуумную энергию при нулевой температуре с больцмановской свободной энергией. Поэтому исследование такой симметрии в моделях теории поля важно, так как она связывает между собой два концептуально различных предела одной и той же теории.

${ }^{*}$ Department of Theoretical Physics, Aristotle University of Thessaloniki, Thessaloniki, Greece. E-mail: voiko@physics.auth.gr 
Как отмечено выше, симметрия обращения температуры задается преобразованием $R \rightarrow 1 / T$. Название "симметрия обращения температуры" [1] не вполне корректно, но оно традиционно используется при описании систем, больцмановская свободная энергия которых (предел при бесконечной температуре) при преобразовании $R \rightarrow 1 / T$ оказывается равной казимировской свободной энергии при нулевой температуре. Рассмотрим, например, четырехмерный суперсимметричный невзаимодействующий ансамбль периодических фермионов и антипериодических бозонов. При высокой температуре свободная энергия такой системы имеет вид

$$
F=-\frac{T^{4}}{\pi^{2}}\left(\frac{\pi^{4}}{45}+\frac{7 \pi^{4}}{360}\right)
$$

в то время как казимировская энергия такого ансамбля равна

$$
E_{\mathrm{o}}=\frac{1}{R^{4} \pi^{2}}\left(\frac{\pi^{4}}{45}+\frac{7 \pi^{4}}{360}\right)
$$

Применяя преобразование $R \rightarrow 1 / T$, получаем, что $F=-E_{\text {о }}$, поскольку система оказывается антисимметричной относительно преобразования обращения температуры.

Отметим сходство этой симметрии с $T$-дуальностью замкнутой бозонной струны [2]. Квадрат полной массы бозонной струны равен

$$
m^{2}=\frac{n^{2}}{R^{2}}+\frac{w^{2} R^{2}}{\alpha^{\prime 2}}+\frac{2}{\alpha^{\prime}}(N+\tilde{N}-2),
$$

где $\alpha^{1 / 2}$ - радиус самодуальности. Отсюда видно, что квадрат полной массы инвариантен при преобразовании $R \rightarrow \alpha^{\prime} / R$ с заменой $n \rightarrow w$. Это преобразование известно как $T$-дуальность бозонной струны и оно связывает пределы $R \rightarrow 0$ и $R \rightarrow \infty$ струнной теории (на самом деле оба этих предела описывают одну и ту же теорию). Это - внутреннее свойство теории струн. Квадрат полной массы содержит возмущения типа Калуцы-Клейна $n^{2} / R^{2}$ вместе с модами намоток $w^{2} R^{2} / \alpha^{\prime 2}$. Моды намоток отсутствуют в полевых теориях, поэтому $T$-дуальность не наблюдается в теоретико-полевых моделях (пределы $R \rightarrow \infty$ и $R \rightarrow 0$ не связаны друг с другом).

Тем не менее масса Калуцы-Клейна периодического бозона при конечной температуре в теории поля имеет вид (при компактифицированной размерности радиуca $R$ )

$$
m_{n, m}^{2}=\frac{4 \pi^{2} n^{2}}{R^{2}}+4 \pi^{2} m^{2} T^{2}
$$

Моды намоток при этом отсутствуют, но возникают “температурные моды намоток" $\sim m^{2} T^{2}$. В некотором роде симметрия обращения температуры может считаться $T$-дуальностью полевой теории. В самом деле, преобразование $R \rightarrow 1 / T$ оставляет инвариантной массу Калуцы-Клейна. 
Уместно отметить еще одно сходство. Свободная энергия замкнутой бозонной струны изменяется при преобразовании температурной дуальности [3] $T \rightarrow T_{\mathrm{c}}^{2} / T$ (тесно связанном с $T$-дуальностью) следующим образом:

$$
\mathcal{F}\left(\frac{T_{\mathrm{c}}^{2}}{T}\right)=\left(\frac{T_{\mathrm{c}}}{T}\right)^{2} \mathcal{F}(T),
$$

где

$$
T_{\mathrm{c}}=\frac{M_{\mathrm{str}}}{2 \pi}=\frac{1}{2 \pi \alpha^{\prime 1 / 2}}
$$

- температура самодуальности (температура Хагедорна), $M_{\mathrm{str}}$ - струнный масштаб. В силу температурной дуальности предел высокой температуры (много выше температуры Хагедорна) связан с пределом низкой температуры (много ниже температуры Хагедорна). Использование преобразования обращения температуры в случае теории поля не приводит ни к каким подобным результатам, поэтому связать высокотемпературные и низкотемпературные пределы не удается. Отметим, однако, что имеет место некоторое сходство с симметрией обращения температуры. В теории поля масштабированная бозонная свободная энергия $f(\xi)$ при $\xi=R T$ ковариантна при преобразовании $\xi \rightarrow 1 / \xi$ и удовлетворяет соотношению

$$
f(\xi)=\xi^{4} f\left(\frac{1}{\xi}\right) .
$$

В силу вышесказанного отметим, что симметрия обращения температуры есть подходящая комбинация струнного преобразования $T$-дуальности и температурной дуальности, проявляющейся в моделях теории поля в компактифицированных размерностях и при конечной температуре.

В общем случае при исследовании четырехмерных моделей с одной компактифицированной координатой получается, что масштабированный эффективный потенциал бозонной частицы при конечной температуре инвариантен относительно преобразования обращения температуры, а эффективный потенциал периодического фермиона и антипериодического бозона, где требуется периодичность граничных условий относительно компактифицированной координаты, оказывается антисимметричным. Более того, вакуумная энергия для периодических бозонов и антипериодических фермионов оказывается симметричной при $R \rightarrow 1 / T$ [1].

Ясно, что граничные условия при компактификации на окружность оказывают влияние на результат преобразования $R \rightarrow 1 / T$, т.е. делают ансамбль симметричным или антисимметричным.

В настоящей работе исследование симметрии обращения температуры расширяется на пятимерные орбифолдные модели. Показано, как орбифолдные граничные условия модифицируют преобразования эффективного потенциала относительно симметрии $R \rightarrow 1 / T$.

Теоретико-полевые орбифолдные компактификации полезны со многих теоретических точек зрения, например в проблеме киральных фермионов в полевых теориях старших размерностей, которая может быть решена с помощью теоретико-полевой 
орбифолдной компактификации [4]-[6], первоначально использованной для компактификаций в полевой теории струн. В описании орбифолдных компактификаций фермионы локализуются на четырехмерных гиперповерхностях, называемых бранами, которые отвечают фиксированным точкам орбифолда. Кривизна в этих точках сингулярна. Таким способом можно удовлетворить условиям киральности. Орбифолды широко применяются в теоретических исследованиях таких, как нарушение калибровочной симметрии [7]-[9] и нарушение суперсимметрий в старших размерностях [6], [7], [10]-[12]. Много альтернативных моделей в размерностях $d=5$ и $d=6$ было введено в основном с использованием структур орбифолдов $S^{1} / Z_{2}$ и $S^{1} / Z_{2} \times Z_{2}^{\prime}$ в качестве дополнительных измерений. Главная задача всех моделей старших размерностей состоит в описании Стандартной модели как низкоэнергетического предела. Одним из перспективных классов таких моделей оказываются так называемые унифицирующие модели калибровочного бозона Хиггса [13], [14], в которых поле Хиггса отождествляется с компонентой калибровочного поля, отвечающей дополнительной координате. В настоящей работе используется калибровочно-полевое описание бозона Хиггса с орбифолдной компактификацией типа $S^{1} / Z_{2}$. Обычно в таких моделях отправной точкой является калибровочная теория в старших размерностях (пятимерная или шестимерная), которая может быть или не быть суперсимметричной для какой-либо простой калибровочной группы, т.е. эта теория не записывается в виде произведения групп, поэтому обладает единственной константой связи. Калибровочная симметрия нарушается введением орбифолдных граничных условий. Дальнейшее нарушение калибровочной симметрии достигается с помощью радиационных поправок с использованием механизма Хосотани [15] (наклон вильсоновской линии), поскольку вакуумное ожидание поля Хиггса пропорционально фазе вильсоновской кривой.

В настоящей работе исследуется эффективный потенциал в объемлющем пространстве для пятимерной $N=1$ суперсимметричной $S U(3)_{\mathrm{c}} \times S U(3)_{\mathrm{w}}$-унифицирующей калибровочной модели бозона Хиггса при конечной температуре [16]. В этой модели на поля налагаются орбифолдно компактифицирующие граничные условия типа $S^{1} / Z_{2}$ и исследуется симметрия обращения температуры [1] $R \rightarrow 1 / T$ для эффективного потенциала в объемлющем пространстве. Применяется условие минимизации эффективного потенциала на вильсоновской линии $\alpha=0,1$ и метод фаз Шерка-Шварца для s-партонов. Далее, набор частиц теории должен совпадать с набором частиц $N=1, d=5$ векторного мультиплета, который в размерности $d=4$ эквивалентен набору частиц $N=2$ суперсимметричного векторного мультиплета [17], [18] (один векторный и один киральный четырехмерный мультиплеты). Кроме того, необходимо добавить поля материи в объемлющем пространстве с различными числами ароматов и представлениями групп, чтобы исследовать их влияние на преобразование обращения температуры в соответствующем эффективном потенциале (оригинальный метод вычисления эффективного функционала был предложен в работе [19]).

В разделе 2 дано краткое описание процедуры орбифолдной компактификации. 


\section{2. КРАТКИЙ ОБЗОР ОРБИФОЛДНОЙ ТЕХНИКИ}

Использование орбифолдной компактификации представляет собой метод, возникший из теории струн, который был сначала применен в физике $\mathrm{TeV}$-энергий для построения киральных фермионов из векторноподобных теорий в старших размерностях [4], [6], [11]. С помощью этого метода строились механизмы нарушения суперсимметрии и калибровочной симметрии в фиксированных точках орбифолдов [7], [8], [10], [12]. Орбифолды получаются при отождествлении относительно несвободного действия дискретной группы $H$ на компактном многообразии $C$. При действии такой группы, описываемым отображением $\zeta_{h}$, точка $y$ компактного многообразия $C$ отождествляется с точкой $\zeta_{h}(y)$, т.е.

$$
y \sim \zeta_{h}(y) .
$$

Лагранжиан полевой теории должен быть при этом инвариантным относительно отождествления (8). Пусть $\phi(x, y)$ - поля теории, $y$ - координата компактного пространства, разбиваемого на орбифолды. Тогда

$$
L(\phi(x, y))=L\left(\phi\left(x, \zeta_{h}(y)\right)\right.
$$

что в терминах полей принимает вид

$$
\phi\left(x, \zeta_{h}(y)\right)=Z_{h} \phi(x, y) .
$$

Преобразование $Z_{h}$ должно быть симметрией лагранжиана, а поля теории должны изменяться таким образом, чтобы эта симметрия имела место. Условие того, что действие группы $H$ на $C$ несвободно, означает, что преобразование $\zeta_{h}$ имеет фиксированные точки. Поэтому результирующее фактор-пространство $O \equiv C / H$ оказывается в фиксированных точках не многообразием, а сингулярным пространством, называемым орбифолдом. Следует отметить, что в случае, если $\phi(x, y)$ есть представление некоторой группы, то $Z_{h}$ может быть нетривиальным представлением дискретной группы $H$. При этом калибровочная симметрия между компонентами калибровочного мультиплета может быть нарушена. В разделе 4 мы опишем пример орбифолда $S^{1} / Z_{2}$, используемого в дальнейшем.

\section{3. НАРУШЕНИЕ СИММЕТРИИ ПО ШЕРКУ-ШВАРЦУ}

В этом разделе описывается механизм Шерка-Шварца [20] и вводятся обозначения, используемые в настоящей работе. Механизм нарушения суперсимметрии по Шерку и Шварцу основан на наложении различных граничных условий на фермионные и бозонные моды при преобразовании $y \rightarrow y+2 \pi R[5]$ :

$$
\Phi(x, y+2 \pi R)=e^{i 2 \pi \beta} \Phi(x, y) .
$$

С учетом этих граничных условий разложения Калуцы-Клейна для полей имеют вид

$$
\Phi(x, y)=\sum_{n=-\infty}^{\infty} e^{i y(n+\beta) / R} \Phi^{n}(x)
$$


или, эквивалентно,

$$
\begin{aligned}
& \Phi(x, y)=\sum_{n=-\infty}^{\infty} \cos \left(\frac{(n+\beta) y}{R}\right) \Phi^{n}(x), \\
& \Phi(x, y)=\sum_{n=-\infty}^{\infty} \sin \left(\frac{(n+\beta) y}{R}\right) \Phi^{n}(x),
\end{aligned}
$$

в случае общего орбифолда.

\section{4. ОРБИФОЛД $S^{1} / Z_{2}$}

Одним из наиболее часто применяемых в теории поля одномерных орбифолдов является орбифолд $S^{1} / Z_{2}$. Он получается при отождествлении точки $y$ на $S^{1}$ с точкой $-y$, т.е. применяется соотношение эквивалентности $Z_{2}$ [21]:

$$
Z_{2}: y \rightarrow-y,
$$

в котором координата на окружности $S^{1}$ есть $-\pi R<y \leqslant \pi R, R$ - радиус окружности. Действие группы $Z_{2}$ имеет две фиксированные точки $y=0$ и $y=\pi R$, называемые бранами в терминах пространства-времени $M^{4} \times S^{1} / Z_{2}$. Фактор-пространство при отождествлении относительно действия $Z_{2}$ оказывается орбифолдом с координатой $0 \leqslant y \leqslant \pi R$. Квантовое поле, которое может служить представлением калибровочной группы, преобразуется под действием $Z_{2}$ следующим образом:

$$
\phi(x,-y)=Z \phi(x, y) .
$$

Легко видеть, что $Z^{2}=I$. Поэтому в пространстве полевых представлений группа $Z$ диагонализуется с собственными значениями \pm 1 . Таким образом, $Z$ может быть либо $I$, либо $-I$, либо смешанной диагональной матрицей $P$ с собственными значениями \pm 1 . В последнем случае $Z$ можно применить для нарушения симметрии (см. ниже).

В дальнейшем орбифолды используются для нарушения калибровочной симметрии. Приведем пример, чтобы показать, как это получается. Рассмотрим чистую пятимерную модель $S U(3)$ с действием

$$
S=\int d x^{4} d y\left(-\frac{1}{4} F^{M N} F_{M N}\right),
$$

где $M, N=1,2,3,4,5$. Для того чтобы нарушить симметрию $S U(3)$, наложим следующие граничные условия на связность калибровочного поля $A_{M}$, принимающую значения в алгебре Ли $S U(3)$ :

$$
A_{M}(x, y)=\Lambda_{M}^{N} P A_{N}(x,-y) P^{\dagger},
$$

где

$$
\Lambda=\left(\begin{array}{ccccc}
1 & 0 & 0 & 0 & 0 \\
0 & 1 & 0 & 0 & 0 \\
0 & 0 & 1 & 0 & 0 \\
0 & 0 & 0 & 1 & 0 \\
0 & 0 & 0 & 0 & -1
\end{array}\right)
$$


что следует из требования пятимерной лоренцевой инвариантности [17], и

$$
P=\left(\begin{array}{ccc}
-1 & 0 & 0 \\
0 & -1 & 0 \\
0 & 0 & 1
\end{array}\right)
$$

В этом частном представлении $P$ группы $Z_{2}$ (заметим, что $P^{2}=1$ ) поля $A_{\mu}^{1,2,3}$ и $A_{\mu}^{8}$ четны, а поля $A_{\mu}^{4,5,6,7}$ нечетны. Таким образом, в фиксированных орбифолдных точках, т.е. на четырехмерном пространстве, группа $S U(3)$ нарушается до $S U(2) \times U(1)$, так как гармоники каждого из полей $A_{\mu}^{4,5,6,7}$ не содержат нулевой моды. Тем самым при энергиях много ниже масштаба компактификации спектр частиц распадается на $S U(2) \times U(1)$-мультиплеты. Заметим, что исходная калибровочная симметрия ограничивается до централизатора представления $P$ в $S U(3)$, или, эквивалентно, калибровочную симметрию нарушают как раз те полевые компоненты, для которых $\left[P, A_{\mu}^{\alpha}\right] \neq 0$. Отметим, что, кроме описанной выше, существует еще одна дискретная симметрия $Z_{2}^{\prime}$ в случае одного дополнительного измерения. Эта дополнительная орбифолдизация использовалась для нарушения суперсимметрии и калибровочной симметрии в некоторых моделях [7], [12], [13].

\section{5. МОДЕЛЬ УНИФИЦИРОВАННОГО КАЛИБРОЧНОГО БОЗОНА ХИГГСА НА $S^{1} / Z_{2}$}

В этом разделе мы рассмотрим пятимерную $S U(N)$-калибровочную теорию на пространстве $S^{1} / Z_{2} \times M^{4}$ (используем обозначения из работы [9]). Калибровочные поля определены на объемлющем пространстве. Пусть $y$ - пятая координата, заданная на окружности $S^{1}$. Орбифолд $S^{1} / Z_{2}$ строится с помощью двух отождествлений координат: $y \rightarrow-y$ и $y-\pi R \rightarrow \pi R-y$, где $R-$ радиус окружности $S^{1}$.

Из формулировки калибровочной теории на $S^{1} / Z_{2}$ следует, что $Z_{2}$ должна быть симметрией лагранжиана. Из орбифолдного отождествления координат и из требования того, что $Z_{2}$ представляет собой симметрию лагранжиана, вытекают следующие законы преобразования полей под действием группы $Z_{2}$ :

$$
\begin{aligned}
A_{\mu}\left(x^{\mu},-y\right) & =P A_{\mu}\left(x^{\mu}, y\right) P^{\dagger}, \\
A_{5}\left(x^{\mu},-y\right) & =-P A_{5}\left(x^{\mu}, y\right) P^{\dagger}, \\
\phi\left(x^{\mu},-y\right) & =\eta T(P) \phi\left(x^{\mu}, y\right), \\
\psi\left(x^{\mu},-y\right) & =\eta^{\prime} T(P) \gamma^{5} \psi\left(x^{\mu}, y\right),
\end{aligned}
$$

где $A_{\mu}$ и $A_{5}$ - калибровочные поля в четырехмерном пространстве и пятая компонента калибровочного поля соответственно. Величина вакуумного ожидания $A_{5}$ в дальнейшем играет роль поля Хиггса. Преобразования (23) и (24) описывают преобразования бозонов и фермионов, $P$ представляет собой подходящее представление группы $Z_{2}$ на компонентах калибровочного поля, а $T(P)$ также задает подходящее представление. Например в случае, если $\psi$ преобразуется по фундаментальному или присоединенному представлению, $T(P) \psi$ отвечает соответственно $P \psi$ и $P \psi P^{\dagger}$. 
Наконец, $\eta$ и $\eta^{\prime}$ принимают значения \pm 1 . Таким же образом можно подставить $P^{\prime}$ вместо $P$ и рассмотреть преобразование $y-\pi R \rightarrow \pi R-y$, при этом будут иметь место те же самые соотношения. В общем случае $P \neq P^{\prime}$, но в настоящей работе рассматривается случай $P=P^{\prime}$. В соответствии со знаками собственных значений $P$, $P^{\prime}$, т.е. $( \pm 1, \pm 1)$, калибровочные поля, например, имеют следующие разложения по гармоникам:

$$
\begin{aligned}
& A_{\mu}\left(x^{\mu}, y\right)_{(+,+)}=\frac{1}{\sqrt{2 \pi R}} \sum_{n=0}^{\infty} A_{\mu}^{(n)}\left(x^{\mu}\right)_{(+,+)} \cos \left(\frac{n y}{R}\right) \\
& A_{\mu}\left(x^{\mu}, y\right)_{(+,-)}=\frac{1}{\sqrt{2 \pi R}} \sum_{n=0}^{\infty} A_{\mu}^{(n)}\left(x^{\mu}\right)_{(+,-)} \cos \left(\frac{(n+1 / 2) y}{R}\right), \\
& A_{\mu}\left(x^{\mu}, y\right)_{(-,+)}=\frac{1}{\sqrt{2 \pi R}} \sum_{n=0}^{\infty} A_{\mu}^{(n)}\left(x^{\mu}\right)_{(-,+)} \sin \left(\frac{(n+1 / 2) y}{R}\right), \\
& A_{\mu}\left(x^{\mu}, y\right)_{(-,-)}=\frac{1}{\sqrt{2 \pi R}} \sum_{n=0}^{\infty} A_{\mu}^{(n)}\left(x^{\mu}\right)_{(-,-)} \sin \left(\frac{(n+1) y}{R}\right) .
\end{aligned}
$$

Оставшиеся поля имеют схожие разложения. Отметим, что только поля с четностями $(+,+)$ имеют нулевые моды, и тем самым только эти поля остаются безмассовыми частицами при энергиях много меньше энергии компактификации. В настоящей работе будем считать, что нулевые моды поля $A_{5}$ имеют ветви $(1,2,1 / 2)$ и $(1,2,-1 / 2)$ относительно действия остаточной калибровочной группы $S U(3)_{\mathrm{c}} \times S U(2)_{\mathrm{L}} \times U(1)_{Y}$ (согласно работе [9]). Эти нулевые моды интерпретируются как дублеты Хиггса, что лежит в основе метода унификации калибровочного поля и бозона Хиггса. Особенно важно то, что с учетом радиационных поправок масса бозона Хиггса оказывается конечной. Это следует из калибровочной инвариантности в пятимерном пространстве и обеспечивает безмассовость компоненты $A_{5}$. В случае пятимерной $N=1$ суперсимметрии после компактификации компонента $A_{5}$ объединяется с присоединенным скалярным полем. $N=1$ суперсимметрия в пятимерном пространстве отвечает $N=2$ суперсимметрии в четырехмерном пространстве, в котором пятимерный векторный мультиплет состоит из четырехмерного векторного и четырехмерного скалярного мультиплетов, лежащих в присоединенном представлении калибровочной группы, т.е.

$$
V_{5}=\left(A^{M}, \lambda, \lambda^{\prime}, \sigma\right)
$$

распадается на

$$
V=\left(A^{\mu}, \lambda\right), \quad \Sigma\left(\sigma+i A_{5}, \lambda^{\prime}\right)
$$

присоединенное векторное и присоединенное четырехмерное киральное суперполя:

$$
\begin{aligned}
& V=-\theta \sigma^{\mu} \bar{\theta} A_{\mu}+i \bar{\theta}^{2} \theta \lambda-i \theta^{2} \bar{\theta} \bar{\lambda}+\frac{1}{2} \bar{\theta}^{2} \bar{\theta}^{2} D, \\
& \Sigma=\frac{1}{\sqrt{2}}\left(\sigma+i A_{5}\right)+\sqrt{2} \theta \lambda^{\prime}+\theta^{2} F .
\end{aligned}
$$


Орбифолдные граничные условия в суперсимметричном случае имеют вид

$$
\begin{aligned}
\left(\begin{array}{c}
V\left(x^{\mu},-y\right) \\
\Sigma\left(x^{\mu},-y\right)
\end{array}\right) & =P\left(\begin{array}{c}
V\left(x^{\mu}, y\right) \\
-\Sigma\left(x^{\mu},-y\right)
\end{array}\right) P^{\dagger}, \\
\left(\begin{array}{c}
V\left(x^{\mu}, \pi R-y\right) \\
\Sigma\left(x^{\mu}, \pi R-y\right)
\end{array}\right) & =P\left(\begin{array}{c}
V\left(x^{\mu}, \pi R+y\right) \\
-\Sigma\left(x^{\mu}, \pi R+y\right)
\end{array}\right) P^{\dagger} .
\end{aligned}
$$

В настоящей работе согласно работе [9] мы добавляем вспомогательные поля материи в объемлющем пространстве (гипермультиплеты в терминах $N=2$ суперсимметрии в размерности 4) в присоединенном и фундаментальном представлениях калибровочной группы, т.е. добавляем $N_{\mathrm{f}} f$ фундаментальных гипермультиплетов и $N_{\alpha}$ присоединенных гипермультиплетов. В размерности $4 N=1$ пятимерные фундаментальный и присоединенный гипермультиплеты (обозначаемые соответственно $\Psi$ и $\Psi^{\alpha}$ ) отвечают четырем $N=1$ киральным и антикиральным суперполям, т.е.

$$
\Psi=\left(\phi, \phi^{\mathrm{c} \dagger}, \widetilde{\phi}, \widetilde{\phi}^{\mathrm{c} \dagger}\right)
$$

распадается до кирального суперполя $\Phi=(\phi, \widetilde{\phi})$ и антикирального суперполя $\Phi^{\mathrm{c}}=\left(\phi^{\mathrm{c}}, \widetilde{\phi}^{\mathrm{c}}\right)$. Также $\Psi^{\alpha}=\left(\phi^{\alpha}, \phi^{\alpha c \dagger}, \widetilde{\phi}^{\alpha}, \widetilde{\phi}^{\alpha c \dagger}\right)$ распадается на киральное суперполе $\Phi=\left(\phi^{\alpha}, \widetilde{\phi}^{\alpha}\right)$ и на антикиральное присоединенное суперполе $\Phi^{\alpha c}=\left(\phi^{\alpha c}, \widetilde{\phi}^{\alpha c}\right)$ в четырехмерном пространстве. Орбифолдные граничные условия для суперполей материи имеют вид

$$
\begin{aligned}
\left(\begin{array}{c}
\Phi\left(x^{\mu},-y\right) \\
\Phi^{\mathrm{c}}\left(x^{\mu},-y\right)
\end{array}\right) & =\eta P\left(\begin{array}{c}
\Phi\left(x^{\mu}, y\right) \\
-\Phi^{c \dagger}\left(x^{\mu}, y\right)
\end{array}\right) \\
\left(\begin{array}{c}
\Phi\left(x^{\mu}, \pi R-y\right) \\
\Phi^{\mathrm{c} \dagger}\left(x^{\mu}, \pi R-y\right)
\end{array}\right) & =\eta^{\prime} P^{\prime}\left(\begin{array}{c}
\Phi\left(x^{\mu}, \pi R+y\right) \\
-\Phi^{\mathrm{c} \dagger}\left(x^{\mu}, \pi R+y\right)
\end{array}\right) \\
\left(\begin{array}{c}
\Phi^{\alpha}\left(x^{\mu},-y\right) \\
\Phi^{\alpha c^{\dagger}}\left(x^{\mu},-y\right)
\end{array}\right) & =\eta P\left(\begin{array}{c}
\Phi^{\alpha}\left(x^{\mu}, y\right) \\
-\Phi^{\alpha{ }^{\dagger}}\left(x^{\mu}, y\right)
\end{array}\right) P^{\dagger}, \\
\left(\begin{array}{c}
\Phi^{\alpha}\left(x^{\mu}, \pi R-y\right) \\
\Phi^{\alpha c^{\dagger}}\left(x^{\mu}, \pi R-y\right)
\end{array}\right) & =\eta^{\prime} P^{\prime}\left(\begin{array}{c}
\Phi^{\alpha}\left(x^{\mu}, \pi R+y\right) \\
-\Phi^{\alpha c^{\dagger}}\left(x^{\mu}, \pi R+y\right)
\end{array}\right) P^{\prime \dagger}
\end{aligned}
$$

В разделах 6, 7 рассматриваются две суперсимметричные модели $S U(3)_{\mathrm{c}} \times S U(3)_{\mathrm{w}}$ и $S U(5)$ в случае $P=P^{\prime}$.

\section{6. КАЛИБРОВОЧНАЯ $S U(3)_{\mathrm{C}} \times S U(3)_{\mathrm{W}}$-МОДЕЛЬ}

Рассмотрим $S U(3)_{\text {c }} \times S U(3)_{\text {w }}$-модель в пятимерном пространстве с представлениями $Z_{2}, P$ и $P^{\prime}$, имеющими в базисе $S U(3)_{\mathrm{w}}$ вид

$$
P=P^{\prime}=\left(\begin{array}{ccc}
1 & 0 & 0 \\
0 & -1 & 0 \\
0 & 0 & -1
\end{array}\right)
$$


Компоненты $V$ и $\Sigma$, описанные в разделе 5 , после преобразований

$$
\begin{aligned}
\left(\begin{array}{c}
V\left(x^{\mu},-y\right) \\
\Sigma\left(x^{\mu},-y\right)
\end{array}\right) & =P\left(\begin{array}{c}
V\left(x^{\mu}, y\right) \\
-\Sigma\left(x^{\mu},-y\right)
\end{array}\right) P^{\dagger} \\
\left(\begin{array}{c}
V\left(x^{\mu}, \pi R-y\right) \\
\Sigma\left(x^{\mu}, \pi R-y\right)
\end{array}\right) & =P\left(\begin{array}{c}
V\left(x^{\mu}, \pi R+y\right) \\
-\Sigma\left(x^{\mu}, \pi R+y\right)
\end{array}\right) P^{\dagger}
\end{aligned}
$$

принимают вид

$$
\begin{gathered}
V=\left(\begin{array}{lll}
(+,+) & (-,-) & (-,-) \\
(-,-) & (+,+) & (+,+) \\
(-,-) & (+,+) & (+,+)
\end{array}\right), \\
\Sigma=\left(\begin{array}{lll}
(-,-) & (+,+) & (+,+) \\
(+,+) & (-,-) & (-,-) \\
(+,+) & (-,-) & (-,-)
\end{array}\right) .
\end{gathered}
$$

Отсюда видно, что симметрия $S U(3)_{\mathrm{w}}$ нарушается до симметрии $S U(2)_{\mathrm{L}} \times U(1)_{Y}$ нулевых мод в секторе $(+,+)$ калибровочного поля $V$. Кроме того, нулевые моды $\Sigma$ обнаруживают структуру дублета Хиггса. Следует отметить, что в этом случае

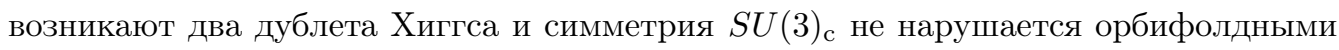
граничными условиями $\left(P=P^{\prime}=I\right.$ в базисе $\left.S U(3)_{\text {c }}\right)$. Теперь вакуумные ожидания скалярной части поля $\Sigma$ оказываются дублетом, так что степени свободы вильсоновской линии, использующие остаточную калибровочную симметрию $S U(2)$, могут быть записаны в виде [19]

$$
\langle\Sigma\rangle=\frac{1}{g R} \sum_{\alpha} \alpha_{\alpha} \frac{\lambda_{\alpha}}{2}=\left(\begin{array}{ccc}
0 & 0 & \alpha \\
0 & 0 & 0 \\
\alpha & 0 & 0
\end{array}\right),
$$

где $g$ - константа связи пятимерной теории. Отметим существование ненулевого вакуумного среднего. Параметр $\alpha$ относится к фазе вильсоновской линии и задает дальнейшее нарушение калибровочной симметрии. В настоящей работе применяются два способа нарушения симметрии, определяемые величиной параметра $\alpha[16]$ :

1) $\alpha=1$, при котором $S U(2)_{\mathrm{L}}$ нарушается до $U^{\prime}(1)$ и остаточная симметрия есть $U^{\prime}(1) \times U(1)_{Y}$;

2) $\alpha=0$, при котором $S U(2)_{\mathrm{L}}$ остается ненарушенной.

Эти два значения соответствуют минимумам эффективного потенциала при нулевой температуре [9].

Помимо калибровочного мультиплета, описанного выше, спектр элементарных частиц теории пополнен дополнительными полями материи, а именно вводятся $N_{\alpha}$ присоединенных и $N_{\text {f }}$ фундаментальных киральных суперполей, описываемых после уравнения (30). Производится дальнейшее разложение полей на классы, т.е. $N_{\alpha}$ распадается на $N_{\alpha}^{+}$и $N_{\alpha}^{-}$, а $N_{\mathrm{f}}-$ на $N_{\mathrm{f}}^{+}$и $N_{\mathrm{f}}^{-}$в соответствии со знаком в произведении $\eta \eta^{\prime}$ (см. уравнение (32) и ниже). $N_{\mathrm{f}}^{+}$и $N_{\alpha}^{+}$отвечают $\eta \eta^{\prime}=1$, а $N_{\alpha}^{-}$ и $N_{\mathrm{f}}^{-}$соответствуют $\eta \eta^{\prime}=-1$ (т.е. в первых двух классах полей оказываются поля с граничными условиями $(+,+)$ и $(-,-))$. 


\section{7. $S U(3)_{\mathrm{C}} \times S U(3)_{\mathrm{W}}$ МОДЕЛЬ ПРИ КОНЕЧНОЙ ТЕМПЕРАТУРЕ}

В этом разделе строится эффективный потенциал при конечной температуре [16], [19] для каждого из мультиплетов, описанных в предыдущих разделах. Полный эффективный потенциал $V_{\text {tot }}$ задается суммой всех вкладов от мультиплетов.

Рассмотрим калибровочный духовой потенциал гаугино $V_{(+)}^{\mathrm{g}}$ (индекс “+” отвечает значению $\eta \eta^{\prime}$ ):

$$
\begin{aligned}
V_{(+)}^{\mathrm{g}}= & -2 \int \frac{d p^{d-2}}{(2 \pi)^{d-2}} \frac{T}{R} \sum_{n, m=-\infty}^{\infty} \ln \left[n^{2}\left(\frac{2 \pi}{R}\right)^{2}+p^{2}+(2 \pi T)^{2} m^{2}\right]+ \\
& +\ln \left[(n-\alpha)^{2}\left(\frac{2 \pi}{R}\right)^{2}+p^{2}+(2 \pi T)^{2} m^{2}\right]+ \\
& +2 \ln \left[\left(n-\frac{\alpha}{2}\right)^{2}\left(\frac{2 \pi}{R}\right)^{2}+p^{2}+(2 \pi T)^{2} m^{2}\right]+ \\
& +2 \int \frac{d p^{d-2}}{(2 \pi)^{d-2}} \frac{T}{R} \sum_{n, m=-\infty}^{\infty} \ln \left[(n-\alpha+\beta)^{2}\left(\frac{2 \pi}{R}\right)^{2}+\right. \\
& \left.+p^{2}+(2 \pi T)^{2}\left(m+\frac{1}{2}\right)^{2}\right]+ \\
& +\ln \left[(n+\beta)^{2}\left(\frac{2 \pi}{R}\right)^{2}+p^{2}+(2 \pi T)^{2}\left(m+\frac{1}{2}\right)^{2}\right]+ \\
& +2 \ln \left[\left(n-\frac{\alpha}{2}+\beta\right)^{2}\left(\frac{2 \pi}{R}\right)^{2}+p^{2}+(2 \pi T)^{2}\left(m+\frac{1}{2}\right)^{2}\right]
\end{aligned}
$$

Здесь и в дальнейшем используется стандартное соглашение для полевых теорий при конечной температуре, т.е. бозоны предполагаются периодическими, а фермионы антипериодическими полями (этот выбор согласован с симметрией Кубо-Мартина-Швингера). В секторе гаугино вводится фаза Шерка-Шварца $\beta$ [19]. Вклад $N_{\mathrm{f}}^{(+)}$ фермионов в фундаментальное представление при $\eta \eta^{\prime}=1$ имеет вид

$$
\begin{aligned}
V_{(+)}^{\mathrm{f}}= & -\frac{4 N_{\mathrm{f}}^{(+)}}{2} \int \frac{d p^{d-2}}{(2 \pi)^{d-2}} \frac{T}{R} \sum_{n, m=-\infty}^{\infty} \ln \left[\left(n-\frac{\alpha}{2}+\beta\right)^{2}\left(\frac{2 \pi}{R}\right)^{2}+p^{2}+(2 \pi T)^{2} m^{2}\right]+ \\
& +\ln \left[(n+\beta)^{2}\left(\frac{2 \pi}{R}\right)^{2}+p^{2}+(2 \pi T)^{2} m^{2}\right]+ \\
& +\frac{4 N_{\mathrm{f}}^{(+)}}{2} \int \frac{d p^{d-2}}{(2 \pi)^{d-2}} \frac{T}{R} \sum_{n, m=-\infty}^{\infty} \ln \left[\left(n+\frac{\alpha}{2}\right)^{2}\left(\frac{2 \pi}{R}\right)^{2}+\right. \\
& \left.+p^{2}+(2 \pi T)^{2}\left(m+\frac{1}{2}\right)^{2}\right]+ \\
& +\ln \left[n^{2}\left(\frac{2 \pi}{R}\right)^{2}+p^{2}+(2 \pi T)^{2}\left(m+\frac{1}{2}\right)^{2}\right]
\end{aligned}
$$


Вклад $N_{\mathrm{f}}^{(-)}$фермионов при $\eta \eta^{\prime}=-1$ равен:

$$
\begin{aligned}
V_{(-)}^{\mathrm{f}}= & -\frac{4 N_{\mathrm{f}}^{(-)}}{2} \int \frac{d p^{d-2}}{(2 \pi)^{d-2}} \frac{T}{R} \sum_{n, m=-\infty}^{\infty} \ln \left[\left(n-\frac{\alpha}{2}+\frac{1}{2}+\beta\right)^{2}\left(\frac{2 \pi}{R}\right)^{2}+\right. \\
& \left.+p^{2}+(2 \pi T)^{2} m^{2}\right]+ \\
& +\ln \left[\left(n+\frac{1}{2}+\beta\right)^{2}\left(\frac{2 \pi}{R}\right)^{2}+p^{2}+(2 \pi T)^{2} m^{2}\right]+ \\
& +\frac{4 N_{\mathrm{f}}^{(-)}}{2} \int \frac{d p^{d-2}}{(2 \pi)^{d-2}} \frac{T}{R} \sum_{n, m=-\infty}^{\infty} \ln \left[\left(n-\frac{\alpha}{2}+\frac{1}{2}\right)^{2}\left(\frac{2 \pi}{R}\right)^{2}+\right. \\
& \left.+p^{2}+(2 \pi T)^{2}\left(m+\frac{1}{2}\right)^{2}\right]+ \\
& +\ln \left[\left(n+\frac{1}{2}\right)^{2}\left(\frac{2 \pi}{R}\right)^{2}+p^{2}+(2 \pi T)^{2}\left(m+\frac{1}{2}\right)^{2}\right] .
\end{aligned}
$$

Отметим различие этих двух вкладов, обусловленное орбифолдными граничными условиями и различными собственными значениями фаз вильсоновских линий. Эффективный потенциал для $N_{\alpha}^{(+)}$присоединенных киральных мультиплетов полей материи при $\eta \eta^{\prime}=1$ имеет вид

$$
\begin{aligned}
V_{(+)}^{\alpha}= & -\frac{4 N_{\alpha}^{(+)}}{2} \int \frac{d p^{d-2}}{(2 \pi)^{d-2}} \frac{T}{R} \sum_{n, m=-\infty}^{\infty} \ln \left[n^{2}\left(\frac{2 \pi}{R}\right)^{2}+p^{2}+(2 \pi T)^{2} m^{2}\right]+ \\
& +\ln \left[(n-\alpha)^{2}\left(\frac{2 \pi}{R}\right)^{2}+p^{2}+(2 \pi T)^{2} m^{2}\right]+ \\
& +2 \ln \left[\left(n-\frac{\alpha}{2}\right)^{2}\left(\frac{2 \pi}{R}\right)^{2}+p^{2}+(2 \pi T)^{2} m^{2}\right]+ \\
& +\frac{4 N_{\alpha}^{(+)}}{2} \int \frac{d p^{d-2}}{(2 \pi)^{d-2}} \frac{T}{R} \sum_{n, m=-\infty}^{\infty} \ln \left[(n-\alpha+\beta)^{2}\left(\frac{2 \pi}{R}\right)^{2}+\right. \\
& \left.+p^{2}+(2 \pi T)^{2}\left(m+\frac{1}{2}\right)^{2}\right]+ \\
& +\ln \left[(n+\beta)^{2}\left(\frac{2 \pi}{R}\right)^{2}+p^{2}+(2 \pi T)^{2}\left(m+\frac{1}{2}\right)^{2}\right]+ \\
& +2 \ln \left[\left(n-\frac{\alpha}{2}+\beta\right)^{2}\left(\frac{2 \pi}{R}\right)^{2}+p^{2}+(2 \pi T)^{2}\left(m+\frac{1}{2}\right)^{2}\right]
\end{aligned}
$$

Заметим, что при $N_{\alpha}^{(+)}=1$ присоединенный киральный мультиплет в совокупности с калибровочным векторным мультиплетом отвечает $N=2$ суперсимметричной 
теории в четырехмерном пространстве. Наконец, вклад $N_{\alpha}^{(-)}$присоединенных киральных полей при $\eta \eta^{\prime}=-1$ записывается в виде

$$
\begin{aligned}
V_{(-)}^{\alpha}= & \frac{4 N_{\alpha}^{(-)}}{2} \int \frac{d p^{d-2}}{(2 \pi)^{d-2}} \frac{T}{R} \sum_{n, m=-\infty}^{\infty} \ln \left[\left(n+\frac{1}{2}\right)^{2}\left(\frac{2 \pi}{R}\right)^{2}+\right. \\
& \left.+p^{2}+(2 \pi T)^{2}\left(m+\frac{1}{2}\right)^{2}\right]+ \\
& +\ln \left[\left(n-\alpha+\frac{1}{2}\right)^{2}\left(\frac{2 \pi}{R}\right)^{2}+p^{2}+(2 \pi T)^{2}\left(m+\frac{1}{2}\right)^{2}\right]+ \\
& +2 \ln \left[\left(n-\frac{\alpha}{2}+\frac{1}{2}\right)^{2}\left(\frac{2 \pi}{R}\right)^{2}+p^{2}+(2 \pi T)^{2}\left(m+\frac{1}{2}\right)^{2}\right]- \\
& -\frac{4 N_{\alpha}^{(-)}}{2} \int \frac{d p^{d-2}}{(2 \pi)^{d-2}} \frac{T}{R} \sum_{n, m=-\infty}^{\infty} \ln \left[\left(n-\alpha+\beta+\frac{1}{2}\right)^{2} \times\right. \\
& \left.\times\left(\frac{2 \pi}{R}\right)^{2}+p^{2}+(2 \pi T)^{2} m^{2}\right]+ \\
& +\ln \left[\left(n+\beta+\frac{1}{2}\right)^{2}\left(\frac{2 \pi}{R}\right)^{2}+p^{2}+(2 \pi T)^{2} m^{2}\right]+ \\
& +2 \ln \left[\left(n-\frac{\alpha}{2}+\beta+\frac{1}{2}\right)^{2}\left(\frac{2 \pi}{R}\right)^{2}+p^{2}+(2 \pi T)^{2} m^{2}\right]
\end{aligned}
$$

Полный эффективный потенциал для приведенного выше ансамбля частиц при конечной температуре имеет вид

$$
V_{\mathrm{tot}}=V_{(+)}^{\mathrm{g}}+V_{(+)}^{\mathrm{f}}+V_{(+)}^{\alpha}+V_{(-)}^{\alpha}+V_{(-)}^{\mathrm{f}} .
$$

7.1. Случай $\alpha=1, \beta=1 / 2$. Случай $\alpha=1, \beta=1 / 2$ представляет особенный интерес, поскольку остающаяся калибровочная симметрия $S U(2)$, "выживающая" при орбифолдном нарушении симметрии, динамически нарушается до $U^{\prime}(1)$ при введении фазы вильсоновской линии в минимуме $\alpha=1$ [15], [16], [19]. Вклад в поля при этом равен

$$
\begin{aligned}
V_{(+)}^{\mathrm{g}}= & -2 \int \frac{d p^{d-2}}{(2 \pi)^{d-2}} \frac{T}{R} \sum_{n, m=-\infty}^{\infty} 2 \ln \left[n^{2}\left(\frac{2 \pi}{R}\right)^{2}+p^{2}+(2 \pi T)^{2} m^{2}\right]+ \\
& +2 \ln \left[\left(n+\frac{1}{2}\right)^{2}\left(\frac{2 \pi}{R}\right)^{2}+p^{2}+(2 \pi T)^{2} m^{2}\right]+ \\
& +2 \int \frac{d p^{d-2}}{(2 \pi)^{d-2}} \frac{T}{R} \sum_{n, m=-\infty}^{\infty} 2 \ln \left[\left(n+\frac{1}{2}\right)^{2}\left(\frac{2 \pi}{R}\right)^{2}+p^{2}+(2 \pi T)^{2}\left(m+\frac{1}{2}\right)^{2}\right]+ \\
& +2 \ln \left[n^{2}\left(\frac{2 \pi}{R}\right)^{2}+p^{2}+(2 \pi T)^{2}\left(m+\frac{1}{2}\right)^{2}\right]
\end{aligned}
$$


для калибровочного поля, духов и гаугино. Для $N_{\mathrm{f}}^{(-)}$фундаментальных фермионов при $\eta \eta^{\prime}=-1$ имеем

$$
\begin{aligned}
V_{(-)}^{\mathrm{f}}= & -\frac{4 N_{\mathrm{f}}^{(-)}}{2} \int \frac{d p^{d-2}}{(2 \pi)^{d-2}} \frac{T}{R} \sum_{n, m=-\infty}^{\infty} \ln \left[\left(n+\frac{1}{2}\right)^{2}\left(\frac{2 \pi}{R}\right)^{2}+p^{2}+(2 \pi T)^{2} m^{2}\right]+ \\
& +\ln \left[n^{2}\left(\frac{2 \pi}{R}\right)^{2}+p^{2}+(2 \pi T)^{2} m^{2}\right]+ \\
& +\frac{4 N_{\mathrm{f}}^{(-)}}{2} \int \frac{d p^{d-2}}{(2 \pi)^{d-2}} \frac{T}{R} \sum_{n, m=-\infty}^{\infty} \ln \left[n^{2}\left(\frac{2 \pi}{R}\right)^{2}+p^{2}+(2 \pi T)^{2}\left(m+\frac{1}{2}\right)^{2}\right]+ \\
& +\ln \left[\left(n+\frac{1}{2}\right)^{2}\left(\frac{2 \pi}{R}\right)^{2}+p^{2}+(2 \pi T)^{2}\left(m+\frac{1}{2}\right)^{2}\right]
\end{aligned}
$$

а для $N_{\mathrm{f}}^{(+)}$фундаментальных фермионов при $\eta \eta^{\prime}=1$ получаем

$$
\begin{aligned}
V_{(+)}^{\mathrm{f}}= & -\frac{4 N_{\mathrm{f}}^{(+)}}{2} \int \frac{d p^{d-2}}{(2 \pi)^{d-2}} \frac{T}{R} \sum_{n, m=-\infty}^{\infty} \ln \left[n^{2}\left(\frac{2 \pi}{R}\right)^{2}+p^{2}+(2 \pi T)^{2} m^{2}\right]+ \\
& +\ln \left[\left(n+\frac{1}{2}\right)^{2}\left(\frac{2 \pi}{R}\right)^{2}+p^{2}+(2 \pi T)^{2} m^{2}\right]+ \\
& +\frac{4 N_{\mathrm{f}}^{(+)}}{2} \int \frac{d p^{d-2}}{(2 \pi)^{d-2}} \frac{T}{R} \sum_{n, m=-\infty}^{\infty} \ln \left[\left(n+\frac{1}{2}\right)^{2}\left(\frac{2 \pi}{R}\right)^{2}+\right. \\
& \left.+p^{2}+(2 \pi T)^{2}\left(m+\frac{1}{2}\right)^{2}\right]+ \\
& +\ln \left[n^{2}\left(\frac{2 \pi}{R}\right)^{2}+p^{2}+(2 \pi T)^{2}\left(m+\frac{1}{2}\right)^{2}\right] .
\end{aligned}
$$

Наконец, потенциал для $N_{\alpha}^{(+)}$присоединенных киральных суперполей при $\eta^{\prime}=1$ запишется в виде

$$
\begin{aligned}
V_{(+)}^{\alpha}= & -\frac{4 N_{\alpha}^{(+)}}{2} \int \frac{d p^{d-2}}{(2 \pi)^{d-2}} \frac{T}{R} \sum_{n, m=-\infty}^{\infty} 2 \ln \left[n^{2}\left(\frac{2 \pi}{R}\right)^{2}+p^{2}+(2 \pi T)^{2} m^{2}\right]+ \\
& +2 \ln \left[\left(n+\frac{1}{2}\right)^{2}\left(\frac{2 \pi}{R}\right)^{2}+p^{2}+(2 \pi T)^{2} m^{2}\right]+ \\
& +\frac{4 N_{\alpha}^{(+)}}{2} \int \frac{d p^{d-2}}{(2 \pi)^{d-2}} \frac{T}{R} \sum_{n, m=-\infty}^{\infty} 2 \ln \left[\left(n+\frac{1}{2}\right)^{2}\left(\frac{2 \pi}{R}\right)^{2}+\right. \\
& \left.+p^{2}+(2 \pi T)^{2}\left(m+\frac{1}{2}\right)^{2}\right]+ \\
& +2 \ln \left[n^{2}\left(\frac{2 \pi}{R}\right)^{2}+p^{2}+(2 \pi T)^{2}\left(m+\frac{1}{2}\right)^{2}\right],
\end{aligned}
$$


а для $N_{\alpha}^{(-)}$присоединенных киральных суперполей при $\eta \eta^{\prime}=-1$

$$
\begin{aligned}
V_{(-)}^{\alpha}= & \frac{4 N_{\alpha}^{(-)}}{2} \int \frac{d p^{d-2}}{(2 \pi)^{d-2}} \frac{T}{R} \sum_{n, m=-\infty}^{\infty} 2 \ln \left[\left(n+\frac{1}{2}\right)^{2}\left(\frac{2 \pi}{R}\right)^{2}+\right. \\
& \left.+p^{2}+(2 \pi T)^{2}\left(m+\frac{1}{2}\right)^{2}\right]+ \\
& +2 \ln \left[n^{2}\left(\frac{2 \pi}{R}\right)^{2}+p^{2}+(2 \pi T)^{2}\left(m+\frac{1}{2}\right)^{2}\right]- \\
& -\frac{4 N_{\alpha}^{(-)}}{2} \int \frac{d p^{d-2}}{(2 \pi)^{d-2}} \frac{T}{R} \sum_{n, m=-\infty}^{\infty} 2 \ln \left[n^{2}\left(\frac{2 \pi}{R}\right)^{2}+p^{2}+(2 \pi T)^{2} m^{2}\right]+ \\
& +2 \ln \left[\left(n+\frac{1}{2}\right)^{2}\left(\frac{2 \pi}{R}\right)^{2}+p^{2}+(2 \pi T)^{2} m^{2}\right]
\end{aligned}
$$

Полный эффективный потенциал $V_{\text {tot }}$ опять имеет вид

$$
V_{\mathrm{tot}}=V_{(+)}^{\mathrm{g}}+V_{(+)}^{\mathrm{f}}+V_{(+)}^{\alpha}+V_{(-)}^{\alpha}+V_{(-)}^{\mathrm{f}}
$$

Применяя представление

$$
\int \frac{d k^{d}}{(2 \pi)^{d}} \ln \left(k^{2}+a^{2}\right)=-\frac{\Gamma(-d / 2)}{(4 \pi)^{d / 2}} a^{d},
$$

можно записать полный вклад в эффективный потенциал от полей материи $N_{\alpha}^{+}$, $N_{\alpha}^{-}, N_{\mathrm{f}}^{+}, N_{\mathrm{f}}^{-}$и от калибровочного гаугино в виде

$$
\begin{aligned}
V_{\mathrm{tot}}= & \frac{2 T}{R}\left(N_{\mathrm{f}}^{-}+N_{\mathrm{f}}^{+}+2+2 N_{\alpha}^{-}+2 N_{\alpha}^{+}\right) \frac{\Gamma((2-d) / 2)}{(4 \pi)^{(d-2) / 2} \times} \\
& \times\left[\sum_{n, m=-\infty}^{\infty}\left((2 \pi T)^{2} m^{2}+\left(n+\frac{1}{2}\right)^{2}\left(\frac{2 \pi}{R}\right)^{2}\right)^{(d-2) / 2}-\right. \\
& \left.-\sum_{n, m=-\infty}^{\infty}\left((2 \pi T)^{2}\left(m+\frac{1}{2}\right)^{2}+n^{2}\left(\frac{2 \pi}{R}\right)^{2}\right)^{(d-2) / 2}\right]+ \\
& +\frac{2 T}{R}\left(N_{\mathrm{f}}^{-}+N_{\mathrm{f}}^{+}+2+2 N_{\alpha}^{-}+2 N_{\alpha}^{+}\right) \frac{\Gamma((2-d) / 2)}{(4 \pi)^{(d-2) / 2}} \times \\
& \times\left[\sum_{n, m=-\infty}^{\infty}\left((2 \pi T)^{2} m^{2}+n^{2}\left(\frac{2 \pi}{R}\right)^{2}\right)^{(d-2) / 2}-\right. \\
& \left.-\sum_{n, m=-\infty}^{\infty}\left((2 \pi T)^{2}\left(m+\frac{1}{2}\right)^{2}+\left(n+\frac{1}{2}\right)^{2}\left(\frac{2 \pi}{R}\right)^{2}\right)^{(d-2) / 2}\right] .
\end{aligned}
$$


Вводя безразмерный параметр $\xi=R T$, получаем

$$
\begin{aligned}
V_{\mathrm{tot}}= & \frac{2 T}{R}\left(N_{\mathrm{f}}^{-}+N_{\mathrm{f}}^{+}+2+2 N_{\alpha}^{-}+2 N_{\alpha}^{+}\right)\left(\frac{2 \pi}{R}\right)^{d-2} \frac{\Gamma((2-d) / 2)}{(4 \pi)^{(d-2) / 2} \times}- \\
& \times\left[\sum_{n, m=-\infty}^{\infty}\left(\xi^{2} m^{2}+\left(n+\frac{1}{2}\right)^{2}\right)^{(d-2) / 2}-\right. \\
& \left.-\sum_{n, m=-\infty}^{\infty}\left(\xi^{2}\left(m+\frac{1}{2}\right)^{2}+n^{2}\right)^{(d-2) / 2}\right]+ \\
& +\frac{2 T}{R}\left(N_{\mathrm{f}}^{-}+N_{\mathrm{f}}^{+}+2+2 N_{\alpha}^{-}+2 N_{\alpha}^{+}\right)\left(\frac{2 \pi}{R}\right)^{d-2} \frac{\Gamma((2-d) / 2)}{(4 \pi)^{(d-2) / 2}} \times \\
& \times\left[\sum_{n, m=-\infty}^{\infty}\left(\xi^{2} m^{2}+n^{2}\right)^{(d-2) / 2}-\right. \\
& \left.-\sum_{n, m=-\infty}^{\infty}\left(\xi^{2}\left(m+\frac{1}{2}\right)^{2}+\left(n+\frac{1}{2}\right)^{2}\right)^{(d-2) / 2}\right] .
\end{aligned}
$$

Воспользуемся двумерной дзета-функцией Эпштейна [22], [23]

$$
Z_{2}\left|\begin{array}{ll}
g_{1} & g_{2} \\
h_{1} & h_{2}
\end{array}\right|\left(a, a_{1}, a_{2}\right)=\sum_{n, m=-\infty}^{\infty}\left(a_{1}\left(n+g_{1}\right)^{2}+a_{2}\left(m+g_{2}\right)^{2}\right)^{-a} e^{2 \pi i\left(n h_{1}+m h_{2}\right)} .
$$

Тогда (51) принимает вид

$$
\begin{aligned}
V_{\mathrm{tot}}= & \frac{2 T}{R}\left(N_{\mathrm{f}}^{-}+N_{\mathrm{f}}^{+}+2+2 N_{\alpha}^{-}+2 N_{\alpha}^{+}\right)\left(\frac{2 \pi}{R}\right)^{d-2} \frac{\Gamma((2-d) / 2)}{(4 \pi)^{(d-2) / 2}} \times \\
& \times\left[Z_{2}\left|\begin{array}{ll}
0 & \frac{1}{2} \\
0 & 0
\end{array}\right|\left(\frac{2-d}{2}, \xi^{2}, 1\right)-Z_{2}\left|\begin{array}{cc}
\frac{1}{2} & 0 \\
0 & 0
\end{array}\right|\left(\frac{2-d}{2}, \xi^{2}, 1\right)\right]+ \\
& +\frac{2 T}{R}\left(N_{\mathrm{f}}^{-}+N_{\mathrm{f}}^{+}+2+2 N_{\alpha}^{-}+2 N_{\alpha}^{+}\right)\left(\frac{2 \pi}{R}\right)^{d-2} \frac{\Gamma((2-d) / 2)}{(4 \pi)^{(d-2) / 2}} \times \\
& \times\left[Z_{2}\left|\begin{array}{ll}
0 & 0 \\
0 & 0
\end{array}\right|\left(\frac{2-d}{2}, \xi^{2}, 1\right)-Z_{2}\left|\begin{array}{cc}
\frac{1}{2} & \frac{1}{2} \\
0 & 0
\end{array}\right|\left(\frac{2-d}{2}, \xi^{2}, 1\right)\right] .
\end{aligned}
$$

Чтобы аналитически продолжить двумерную дзета-функцию Эпштейна для значений $\operatorname{Re} a<1$, применяется функциональное уравнение

$$
\begin{aligned}
Z_{2}\left|\begin{array}{ll}
g_{1} & g_{2} \\
h_{1} & h_{2}
\end{array}\right|\left(a, a_{1}, a_{2}\right)= \\
=\left(a_{1} a_{2}\right)^{-1 / 2} \pi^{2 a-1} \frac{\Gamma(1-a)}{\Gamma(a)} \exp \left[-2 \pi i\left(g_{1} h_{1}+g_{2} h_{2}\right)\right] \times \\
\quad \times Z_{2}\left|\begin{array}{cc}
h_{1} & h_{2} \\
-g_{1} & -g_{2}
\end{array}\right|\left(1-a, \frac{1}{a_{1}}, \frac{1}{a_{2}}\right) .
\end{aligned}
$$


После некоторых преобразований $V_{\text {tot }}$ принимает вид

$$
\begin{aligned}
V_{\mathrm{tot}}= & 2 \pi^{-d / 2}\left(N_{\mathrm{f}}^{-}+N_{\mathrm{f}}^{+}+2+2 N_{\alpha}^{-}+2 N_{\alpha}^{+}\right) \xi^{d} \frac{\Gamma(d / 2)}{R^{d}} \times \\
& \times\left[Z_{2}\left|\begin{array}{cc}
0 & 0 \\
0 & -\frac{1}{2}
\end{array}\right|\left(\frac{d}{2}, 1, \xi^{2}\right)-Z_{2}\left|\begin{array}{cc}
0 & 0 \\
-\frac{1}{2} & 0
\end{array}\right|\left(\frac{d}{2}, 1, \xi^{2}\right)\right]+ \\
& +2 \pi^{-d / 2}\left(N_{\mathrm{f}}^{-}+N_{\mathrm{f}}^{+}+2+2 N_{\alpha}^{-}+2 N_{\alpha}^{+}\right) \xi^{d} \frac{\Gamma(d / 2)}{R^{d}} \times \\
& \times\left[Z_{2}\left|\begin{array}{cc}
0 & 0 \\
0 & 0
\end{array}\right|\left(\frac{d}{2}, 1, \xi^{2}\right)-Z_{2}\left|\begin{array}{cc}
0 & 0 \\
-\frac{1}{2} & -\frac{1}{2}
\end{array}\right|\left(\frac{d}{2}, 1, \xi^{2}\right)\right] .
\end{aligned}
$$

Теперь положим

$$
\begin{aligned}
& f(\xi)=\xi^{d}\left[Z_{2}\left|\begin{array}{cc}
0 & 0 \\
0 & -\frac{1}{2}
\end{array}\right|\left(\frac{d}{2}, 1, \xi^{2}\right)-Z_{2}\left|\begin{array}{cc}
0 & 0 \\
-\frac{1}{2} & 0
\end{array}\right|\left(\frac{d}{2}, 1, \xi^{2}\right)\right], \\
& g(\xi)=\xi^{d}\left[Z_{2}\left|\begin{array}{ll}
0 & 0 \\
0 & 0
\end{array}\right|\left(\frac{d}{2}, 1, \xi^{2}\right)-Z_{2}\left|\begin{array}{cc}
0 & 0 \\
-\frac{1}{2} & -\frac{1}{2}
\end{array}\right|\left(\frac{d}{2}, 1, \xi^{2}\right)\right] .
\end{aligned}
$$

Имеем

$$
f\left(\frac{1}{\xi}\right)=\frac{1}{\xi^{d}}\left[Z_{2}\left|\begin{array}{cc}
0 & 0 \\
0 & -\frac{1}{2}
\end{array}\right|\left(\frac{d}{2}, 1, \frac{1}{\xi^{2}}\right)-Z_{2}\left|\begin{array}{cc}
0 & 0 \\
-\frac{1}{2} & 0
\end{array}\right|\left(\frac{d}{2}, 1, \frac{1}{\xi^{2}}\right)\right],
$$

или, эквивалентно,

$$
f\left(\frac{1}{\xi}\right)=Z_{2}\left|\begin{array}{cc}
0 & 0 \\
0 & -\frac{1}{2}
\end{array}\right|\left(\frac{d}{2}, \xi^{2}, 1\right)-Z_{2}\left|\begin{array}{cc}
0 & 0 \\
-\frac{1}{2} & 0
\end{array}\right|\left(\frac{d}{2}, \xi^{2}, 1\right)
$$

Из (58) вытекает, что

$$
\begin{aligned}
& Z_{2}\left|\begin{array}{cc}
0 & 0 \\
0 & -\frac{1}{2}
\end{array}\right|\left(\frac{d}{2}, \xi^{2}, 1\right)-Z_{2}\left|\begin{array}{cc}
0 & 0 \\
-\frac{1}{2} & 0
\end{array}\right|\left(\frac{d}{2}, \xi^{2}, 1\right)= \\
& \quad=\left[Z_{2}\left|\begin{array}{cc}
0 & 0 \\
0 & -\frac{1}{2}
\end{array}\right|\left(\frac{d}{2}, 1, \xi^{2}\right)-Z_{2}\left|\begin{array}{cc}
0 & 0 \\
-\frac{1}{2} & 0
\end{array}\right|\left(\frac{d}{2}, 1, \xi^{2}\right)\right]
\end{aligned}
$$

Объединяя теперь уравнения (55), (57) и (59), получаем

$$
f(\xi)=-\xi^{d} f\left(\frac{1}{\xi}\right)
$$

и, аналогично,

$$
g(\xi)=\xi^{d} f\left(\frac{1}{\xi}\right)
$$


Таким образом, полный эффективный потенциал $V_{\text {tot }}$ можно записать в виде

$$
C_{1} V_{\text {tot }}=f(\xi)+g(\xi)
$$

где

$$
C_{1}=\frac{R^{d}}{2 \pi^{-d / 2}\left(N_{\mathrm{f}}^{-}+N_{\mathrm{f}}^{+}+2+2 N_{\alpha}^{-}+2 N_{\alpha}^{+}\right) \Gamma(d / 2)} .
$$

Заметим, что в полном эффективном потенциале симметрия относительно обращения температуры теряется, и он содержит полностью симметричную составляющую $g(\xi)$ и полностью антисимметричную составляющую $f(\xi)$.

7.2. Случай $\alpha=0, \beta=1 / 2$. Рассмотрим теперь случай $\alpha=0, \beta=1 / 2$. Этот случай, однако, не столь интересен с феноменологической точки зрения, поскольку симметрия $S U(2)_{\mathrm{L}}$ остается ненарушенной. Несмотря на это приведем здесь результаты, чтобы увидеть, сохраняется ли симметрия относительно обращения температуры в этом случае. С помощью метода, развитого в п. 7.1, получаем полный эффективный потенциал

$$
\begin{aligned}
V_{\mathrm{tot}}= & 2 \pi^{-d / 2}\left(2 N_{\mathrm{f}}^{+}\right) \xi^{d} \frac{\Gamma(d / 2)}{R^{d}} \times \\
& \times\left[Z_{2}\left|\begin{array}{cc}
0 & 0 \\
0 & -\frac{1}{2}
\end{array}\right|\left(\frac{d}{2}, 1, \xi^{2}\right)-Z_{2}\left|\begin{array}{cc}
0 & 0 \\
-\frac{1}{2} & 0
\end{array}\right|\left(\frac{d}{2}, 1, \xi^{2}\right)\right]+ \\
& +2 \pi^{-d / 2}\left(2 N_{\mathrm{f}}^{-}+4+4 N_{\alpha}^{-}+4 N_{\alpha}^{+}\right) \xi^{d} \frac{\Gamma(d / 2)}{R^{d}} \times \\
& \times\left[Z_{2}\left|\begin{array}{cc}
0 & 0 \\
0 & 0
\end{array}\right|\left(\frac{d}{2}, 1, \xi^{2}\right)-Z_{2}\left|\begin{array}{cc}
0 & 0 \\
-\frac{1}{2} & -\frac{1}{2}
\end{array}\right|\left(\frac{d}{2}, 1, \xi^{2}\right)\right] .
\end{aligned}
$$

Легко доказать, что потенциал

$$
\begin{aligned}
V_{\mathrm{A}}= & 2 \pi^{-d / 2}\left(2 N_{\mathrm{f}}^{+}\right) \xi^{d} \frac{\Gamma(d / 2)}{R^{d}} \times \\
& \times\left[Z_{2}\left|\begin{array}{cc}
0 & 0 \\
0 & -\frac{1}{2}
\end{array}\right|\left(\frac{d}{2}, 1, \xi^{2}\right)-Z_{2}\left|\begin{array}{cc}
0 & 0 \\
-\frac{1}{2} & 0
\end{array}\right|\left(\frac{d}{2}, 1, \xi^{2}\right)\right]
\end{aligned}
$$

антисимметричен относительно инверсии температуры, а потенциал

$$
\begin{aligned}
V_{\mathrm{S}}= & +2 \pi^{-d / 2}\left(2 N_{\mathrm{f}}^{-}+4+4 N_{\alpha}^{-}+4 N_{\alpha}^{+}\right) \xi^{d} \frac{\Gamma(d / 2)}{R^{d}} \times \\
& \times\left[Z_{2}\left|\begin{array}{ll}
0 & 0 \\
0 & 0
\end{array}\right|\left(\frac{d}{2}, 1, \xi^{2}\right)-Z_{2}\left|\begin{array}{cc}
0 & 0 \\
-\frac{1}{2} & -\frac{1}{2}
\end{array}\right|\left(\frac{d}{2}, 1, \xi^{2}\right)\right]
\end{aligned}
$$

симметричен при инверсии температуры. Сравнивая случаи $\alpha=0$ и $\alpha=1$, можно видеть, что в первом случае только фундаментальные фермионы с четностями $(+,+)$ или $(-,-)$ нарушают симметрию обращения температуры, а во втором случае поля всех частиц содержат как симметричную, так и антисимметричную составляющую. Простой анализ показывает, что в силу орбифолдных граничных условий, 
накладываемых на компоненты мультиплета фундаментальных фермионов, симметрия обращения температуры не выполняется в эффективном потенциале, если только он получает вклады от фермионов с четностями $(+,+)$ или $(-,-)$. Этот результат представляет интерес, поскольку в некоторых моделях с дополнительными измерениями все материальные фермионы (фермионы ароматов), т.е. лептоны и кварки локализуются в фиксированных точках орбифолдов и не содержат ни мод Калуцы-Клейна, ни нулевых мод. Наш результат указывает на то, что в моделях с фундаментальными фермионами, локализованными в фиксированных точках орбифолдов, симметрия обращения температуры должна соблюдаться в эффективном потенциале в объемлющем пространстве (в этом случае в обозначениях из предыдущих вычислений $\alpha=0$ и $\beta=1 / 2$ ).

В заключение отметим, что наш выбор параметров $\alpha$ и $\beta$, т.е. $\alpha=0,1$, был наиболее естественным, так как при этих значениях полный эффективный потенциал $V_{\text {tot }}$ достигает своего минимума, а калибровочная симметрия или нарушена (при $\alpha=1$ ), или сохраняется (при $\alpha=0$ ) [9]. Выбор $\beta=1 / 2$ соответствует полной периодичности или полной антипериодичности при повороте $y \rightarrow y+2 \pi R$ [24]. Нас также интересовали прежде всего феноменологически зна́чимые случаи (например, присваивание фазы Шерка-Шварца калибровочным бозонам было бы феноменологически неприемлемо).

Разумеется в моделях калибровочной унификации бозона Хиггса всегда возникает вопрос: какие значения $\alpha$ и $\beta$ задают корректное нарушение симметрии электрослабых взаимодействий. Значения, используемые в настоящей работе, не приводят к корректному нарушению этой симметрии. Численные расчеты, проделанные в работах [9], [16], дают вполне согласованные результаты. В наших исследованиях для нарушения калибровочной симметрии использовались наиболее очевидные величины параметров (минимальные значения эффективного потенциала), а для нарушения суперсимметрии - свойства периодичности и антипериодичности при вращениях $y \rightarrow y+2 \pi R$.

\section{8. ЗАКЛЮЧЕНИЕ}

Дуальности играют важную роль, особенно в эффективных полевых теориях с предсказаниями, которые зна́чимы при энергиях выше экспериментально достижимых в настоящее время или недоступны исследованиям в рамках теории возмущений. В настоящей работе исследовалась дуальность относительно преобразования обращения температуры $R \rightarrow 1 / T$ [1], которая связывает между собой больцмановскую свободную энергию с вакуумной энергией системы при нулевой температуре. Анализ проводился для суперсимметричной пятимерной $S U(3)_{\mathrm{w}} \times S U(3)_{\mathrm{c}}$-модели калибровочной унификации бозона Хиггса. Используемое значение фазы ШеркаШварца, нарушающей симметрию, было выбрано равным $\beta=1 / 2$, а значение фазы вильсоновской линии было выбрано равным $\alpha=0,1$. Выбранное значение $\beta$ отвечает периодичности или антипериодичности полей при преобразовании $y \rightarrow y+2 \pi R[5]$, а значения $\alpha=0,1$ отвечают минимумам эффективного потенциала при нулевой 
температуре [9]. При $\alpha=1$ найдено, что независимо от числа гипермультиплетов полный эффективный потенциал $V_{\text {tot }}$ содержит как антисимметричную составляющую при преобразовании $R \rightarrow 1 / T$ (или, эквивалентно, $\xi \rightarrow 1 / \xi$ при $\xi=R T$ ), выражаемую в терминах $f(\xi)$ следующим образом:

$$
f(\xi)=-\xi^{d} f\left(\frac{1}{\xi}\right),
$$

так и симметричную составляющую

$$
g(\xi)=\xi^{d} f\left(\frac{1}{\xi}\right)
$$

При этом

$$
C_{1} V_{\text {tot }}=f(\xi)+g(\xi)
$$

где $C_{1}$ - константа. В этих формулах $\xi=R T$, где $R$ и $T$ - радиус компактификации и температура системы соответственно. В случае $\alpha=1$ остающаяся после орбифолдной компактификации калибровочная симметрия $S U(2)_{\mathrm{L}}$ нарушена до $U^{\prime}(1)$. Случай $\alpha=0$ отвечает ненарушенной калибровочной симметрии $S U(2)_{\mathrm{L}}$. В этом случае было обнаружено интересное явление. Было показано, что вклад фермионов в фундаментальное представление калибровочной группы с $Z_{2}$ четностями $(+,+)$ или $(-,-)$ оказывается единственным вкладом в эффективный потенциал, который не инвариантен при инверсии температуры. Таким образом, в моделях с фундаментальными фермионами, локализованными в фиксированных точках орбифолдов (т.е. в отсутствие фермионов в объемлющем пространстве), эффективный потенциал в объемлющем пространстве оказывается инвариантным при преобразовании обращения температуры. Этот вывод был проверен для всех возможных случаев, в том числе и в случае, когда фундаментальным s-частицам присваивался ненулевой параметр Шерка-Шварца. Он оказался справедливым во всех этих случаях.

Отметим, наконец, что правильное нарушение электрослабой симметрии требует, чтобы параметр $\alpha$ принимал дробные значения. Исследование таких систем было проведено в работах [9], [16]. Однако при таких значениях симметрия полностью нарушается.

Представляется вполне вероятным, что орбифолдизация оказывает влияние на свойства симметрии эффективного потенциала при преобразованиях обращения температуры, приводя к теоретико-полевой системе, квантуемой на орбифолде. Можно ожидать, что это же свойство будет справедливо для любого ансамбля, квантуемого на многообразии произведения с твистованными граничными условиями. Тем самым специфика граничных условий определяет будет выполнена симметрия или нет.

Благодарности. Автор выражает благодарность Н. Тракасу, С. Массену, Х. Мустакидису и Д. Яковиду. 


\section{Список литературы}

[1] C. Wotzasek, J. Phys. A, 23:9 (1990), 1627-1632; F. C. Santos, A. C. Tort, Phys. Lett. B, 482:1-3 (2000), 323-328; Duality transformations and temperature inversion symmetry, arXiv: hep-th/0110012; A. C. Aguiar Pinto, T. M. Britto, F. Pascoal, F.S. S. da Rosa, Phys. Rev. D, 67:10 (2003), 107701; F. Ravndal, D. Tollefsen, Phys. Rev. D, 40:12 (1989), 4191-4192; A. Gundersen, F. Ravndal, Ann. Phys., 182:1 (1988), 90-111; L.S. Brown, G. J. Maclay, Phys. Rev. D, 184:5 (1969), 1272-1279; V. K. Oikonomou, J. Phys. A, 40:21 (2007), 5725-5731; J. S. Dowker, Class. Quant. Grav., 20:8 (2003), L105-L113.

[2] J. Polchinski, String Theory, V. 1, 2, Cambridge Monogr. Math. Phys., Cambridge Univ. Press, Cambridge, 1998; S. Chaudhuri, Phys. Rev. Lett., 86 (2001), 1943-1946; E. Alvarez, M. A. R. Osorio, Phys. Rev. D, 40 (1989), 1150-1152.

[3] K. R. Dienes, M. Lennek, Phys. Rev. D, 70:12 (2004), 126005.

[4] M. Quiros, Introduction to extra dimensions, arXiv: hep-ph/0606153; New ideas in symmetry breaking, arXiv: hep-ph/0302189; Finite temperature field theory and phase transitions, arXiv: hep-ph/9901312.

[5] I. Antoniadis, S. Dimopoulos, A. Pomarol, M. Quirós, Nucl. Phys. B, 544:3 (1999), 503-519.

[6] A. Delgado, A. Pomarol, M. Quirós, Phys. Rev. D, 60:9 (1999), 095008.

[7] A. Hebecker, J. M. Russell, Nucl. Phys. B, 625:1-2 (2002), 128-150.

[8] A. Hebecker, J. M. Russell, Nucl. Phys. B, 613:1-2 (2001), 3-16.

[9] N. Haba, Y. Hosotani, Y. Kawamura, Progr. Theoret. Phys., 111:2 (2004), 265-289; N. Haba, M. Harada, Y. Hosotani, Y. Kawamura, Nucl. Phys. B, 657:1-3 (2003), 169-213.

[10] Y. Kawamura, Progr. Theoret. Phys., 105:4 (2001), 691-696.

[11] A. Pomarol, M. Quirós, Phys. Lett. B, 438:3-4 (1998), 255-260.

[12] R. Barbieri, L. J. Hall, Y. Nomura, Phys. Rev. D, 63:10 (2001), 105007.

[13] L. J. Hall, Y. Nomura, D. R. Smith, Nucl. Phys. B, 639:1-2 (2002), 307-330; G. Burdman, Y. Nomura, Nucl. Phys. B, 656:1-2 (2003), 3-22.

[14] N. Haba, Y. Hosotani, Y. Kawamura, T. Yamashita, Phys. Rev. D, 70:1 (2004), 015010; Y. Hosotani, S. Noda, K. Takenaga, Phys. Lett. B, 607:3-4 (2005), 276-285; N. Haba, K. Takenaga, T. Yamashita, Phys. Rev. D, 71:2 (2005), 025006; N. Maru, K. Takenaga, Phys. Rev. D, 72:4 (2005), 046003; M. Sakamoto, K. Takenaga, Phys. Rev. D, 76 (2007), 085016; arXiv: 0706.0071; C. A. Scrucca, M. Serone, L. Silvestrini, Nucl. Phys. B, 669:1-2 (2003), 128-158; C. A. Scrucca, M. Serone, A. Wulzer, L. Silvestrini, JHEP, 02 (2004), 049; G. Panico, M. Serone, JHEP, 05 (2005), 024; "The electroweak phase transition in models with gauge-Higgs unification", arXiv: hep-ph/0511348; G. Panico, M. Serone, A. Wulzer, Nucl. Phys. B, 739:1-2 (2006), 186-207; M. Serone, "The Higgs boson as a Gauge field in extra dimensions", 17th Italian Meeting on High Energy Physics, AIP Conf. Proc., 794, no. 1, eds. A. Tricomi, S. Albergo, and M. Chiorboli, Amer. Inst. Phys., Melville, N. Y., 2005, 139-142; arXiv: hep-ph/0508019; C. A. Scrucca, M. Serone, L. Silvestrini, F. Zwirner, Phys. Lett. B, 525:1-2 (2002), 169-174; C. Biggio, M. Quirós, Nucl. Phys. B, 703:1-2 (2004), 199-216.

[15] Y. Hosotani, Phys. Lett. B, 126:5 (1983), 309-313; Ann. Physics, 190:2 (1989), 233-253.

[16] N. Maru, K. Takenaga, Phys. Rev. D, 74:1 (2006), 015017; N. Maru, K. Takenaga, Phys. Lett. B, 637:4-5 (2006), 287-294.

[17] N. Arkani-Hamed, T. Gregoire, J.G. Wacker, JHEP, 03 (2002), 055; arXiv: hep-th/0101233.

[18] L. Alvarez-Gaumé, S.F. Hassan, Fortschr. Phys., 45:3-4 (1997), 159-236; arXiv: hep-th/9701069.

[19] N. Haba, T. Yamashita, JHEP, 02 (2004), 059; arXiv: hep-ph/0401185.

[20] J. Scherk, J. H. Schwarz, Phys. Lett. B, 82:1 (1979), 60-64; Nucl. Phys. B, 153:1-2 (1979), 61-88. 
[21] K. Hwang, J.E. Kim, Orbifold Compactification and Related Phenomenology, arXiv: hep-ph/0411286.

[22] E. Elizalde, A. Romeo, Rev. Math. Phys., 01:1 (1989), 113-128; E. Elizalde, J. Phys. A, 39:21 (2006), 6299-6307; Ten Physical Applications of Spectral Zeta Functions, Lecture Notes in Phys. New Ser. Monogr., 35, Springer, Berlin, 1995; J. Math. Phys., 35:11 (1994), 6100-6122.

[23] Yu. P. Goncharov, A. A. Bytsenko, Nucl. Phys. B, 271:3-4 (1986), 726-748.

[24] I. Antoniadis, C. Muñoz, M. Quirós, Nucl. Phys. B, 397:3 (1993), 515-538.

Поступила в редакцию 30.06.2008 